\title{
Blood Vessels Form a Scaffold for Neuroblast Migration in the Adult Olfactory Bulb
}

\author{
Serena Bovetti, ${ }^{1,2}$ Yi-Chun Hsieh, ${ }^{2}$ Patrizia Bovolin, ${ }^{1}$ Isabelle Perroteau, ${ }^{1}$ Toida Kazunori, ${ }^{3}$ and Adam C. Puche ${ }^{2}$ \\ ${ }^{1}$ Department of Human and Animal Biology, University of Torino, 10123 Torino, Italy, ${ }^{2}$ Department of Anatomy and Neurobiology, University of Maryland, \\ Baltimore, Maryland 21201, and '3Department of Anatomy and Cell Biology, Institute of Health Biosciences, University of Tokushima Graduate School, \\ Tokushima 770-8503, Japan
}

\begin{abstract}
New cells are continuously added to the rodent olfactory bulb $(\mathrm{OB})$, throughout development and in adults. These cells migrate tangentially from the subventricular zone along the rostral migratory stream to the $\mathrm{OB}$, where they migrate radically from the center to periphery of the $\mathrm{OB}$. Although different modalities of radial migration have been described in other brain regions, the mechanisms governing radial migration in the $\mathrm{OB}$ are still mostly unknown. Here, we identify a new modality of migration in which neuronal precursors migrate along blood vessels toward their destination. Our results show that half of the radially migrating cells associate with the vasculature in the granule cell layer of the $\mathrm{OB}$, and in vivo time-lapse imaging demonstrates that they use blood vessels as a scaffold for their migration through an interaction with the extracellular matrix and perivascular astrocyte end feet. The present data provide evidence that a new modality of migration, vasophilic migration, is occurring in the adult brain and reveals a novel role of brain vasculature.
\end{abstract}

Key words: radial migration; vascular; endothelial cell; time lapse; neuronal progenitor; stem cell

\section{Introduction}

The migration of immature neurons from germinal zones to specific positions is a critical step in the development of the brain. Different mechanisms of neuronal migration have been described in the developmental and adult CNS. During the development of different brain structures such as the cortex, cerebellum, and hippocampus, neurons use radial glia processes as scaffold to their destination (Gasser and Hatten, 1990a,b; Wichterle et al., 1997). This glia-dependent radial migration, also called gliophilic migration, is prominent in the embryonic and early postnatal CNS. However, other types of migration independent of radial glia have been described. Neurophilic migration describes the movement of cells using the axons of already differentiated neurons to reach their final location. Homophilic migration involves the movement of neuroblasts using each other as a substrate forming long chains of cells, which was first described in the rostral migratory stream (RMS) of adult rodent, where cells originating from the subventricular zone migrate tangentially along the RMS to reach the olfactory bulb (OB) (Lois et al., 1996). In the $\mathrm{OB}$, the cells detach from chains and disperse radially in the $\mathrm{OB}$. Little is known about the mechanisms governing radial dispersion of neuroblasts in the adult OB.

Radial glia are present in the developing olfactory bulb and are likely to be important for early radial migration. However, mi-

Received June 5, 2006; revised April 16, 2007; accepted April 19, 2007.

This work was supported by National Institute on Deafness and Other Communication Disorders Grant DC05739 to A.C.P. S.B. is the recipient of a fellowship sponsored by Fondazione Cassa di Risparmio di Cuneo. We also thank Dr. Gabor Szabo for use of the GAD65-GFP transgenic mice.

Correspondence should be addressed to Dr. Adam C. Puche, Department of Anatomy and Neurobiology, School of Medicine, University of Maryland, Penn Street, Baltimore, MD 21201. E-mail: apuche@umaryland.edu. DOI:10.1523/JNEUROSCI.0678-07.2007

Copyright $\odot 2007$ Society for Neuroscience $\quad$ 0270-6474/07/275976-05\$15.00/0 gration of immature neurons in the $\mathrm{OB}$ is not limited to development, but continues in adults, when radial glia have disappeared. Previous studies reported the tight association of migrating neuroblasts with blood vessels in the early postnatal and adult RMS, which was hypothesized to be involved in cell survival (Peretto et al., 2005; Yang et al., 2005). We hypothesized that adult neuroblasts may use blood vessels as a scaffold in the absence of radial glia. In this study, we observed that, after reaching the $\mathrm{OB}$, a large number of neuronal precursors associated with the blood vessels and migrated radially into the $\mathrm{OB}$ after these vessels. This novel migratory mechanism, the vasophilic neuronal migration, is involved in neuronal movement into the $\mathrm{OB}$.

\section{Materials and Methods}

Dye labeling. Stereotaxic injections of Cell Tracker green CMFDA (CTG; Invitrogen, Eugene, OR), fluorogold (FG; Invitrogen), or cholera toxin $\beta$-subunit (CTb; Sigma, St. Louis, MO) were performed after our previously described protocols (De Marchis et al., 2001) on CD1-strain postnatal day 21 (P21) mice (Charles River Laboratories, Wilmington, MA). All of the experimental procedures were performed in accordance with protocols approved by our institutional animal care and use committees. Animals were anesthetized by intraperitoneal injection of sodium pentobarbital $(4-5 \mathrm{mg} / 100 \mathrm{mg}$ body weight in $0.9 \%$ saline solution) and $70-90 \mathrm{nl}$ of CTG or $100 \mathrm{nl}$ of FG/CTb injected at stereotaxic coordinates of bregma $+0.5 \mathrm{~mm}$, lateral $\pm 1.0 \mathrm{~mm}$, and $2 \mathrm{~mm}$ deep, corresponding to the anterior subventricular zone (SVZa).

Vessel-neuroblast analysis. Three days after CTG injection, P24 mice were anesthetized and perfused transcardially with $0.9 \%$ saline followed by $5 \mathrm{ml}$ of $1 \%$ and then $5 \mathrm{ml}$ of $5 \%$ porcine type-A gelatin containing 1 $\mathrm{mg} / 5 \mathrm{ml}$ bovine serum albumin tetramethyl-rhodamine isothiocyanate (BSA-rhodamine; Sigma). Immunohistochemical analysis for glial acidic fibrillary protein (GFAP, 1:5000; Dako, High Wycombe, UK) and antiNeuN (1:1000; Millipore, Temecula, CA) were performed on freefloating serial $40 \mu \mathrm{m}$ coronal sections (see supplemental material, avail- 
able at www.jneurosci.org). Fluorescent images for anatomical analysis were captured on an Olympus (Tokyo, Japan) FluoView 500 confocal microscope and the distance between cells and blood vessels, as well as vessels orientation, was calculated using Neurolucida software (see supplemental material, available at www.jneurosci.org). Data are presented as mean \pm SEM or as a cell frequency versus distance histogram [significance determined by multivariate ANOVA (MANOVA)].

Lineage analysis. Fluorogold injections were performed as above and $30 \mathrm{~d}$ after injection, mice were anesthetized and transcardially perfused with $0.9 \%$ saline followed by $4 \%$ paraformaldehyde in $0.1 \mathrm{~m}$ phosphate buffer (PB), pH 7.4. Tissue processing and immunohistochemistry was performed as described above.

Electron microscopy. Three days after CTb injection, P24 mice were anesthetized and perfused transcardially with $0.9 \%$ saline followed by $4 \%$ electron microscopy (EM)-grade paraformaldehyde and 1\% EM-grade gluteraldehyde in $0.1 \mathrm{M} \mathrm{PB}, \mathrm{pH}$ 7.4. The brains were postfixed, sectioned $(25 \mu \mathrm{m})$, incubated with goat anti-CTb (1:5000; List Biologicals, Campbell, CA), and reacted with diaminobenzidine (DAB; $0.5 \mathrm{mg} / \mathrm{ml}$ ), $\mathrm{H}_{2} \mathrm{O}_{2}$ $(1.2 \% \mathrm{v} / \mathrm{v}), 0.2 \% \mathrm{NiCl}$, and $0.25 \% \mathrm{CoCl}$ (Adams, 1981). The DABstained sections were further processed for electron microscopy by infiltration with resin following our previously published protocols (Toida et al., 1998) (see supplemental material, available at www.jneurosci.org).

Time-lapse confocal microscopy. CTG was injected into P21 mice and after $3 \mathrm{~d}$ mice were perfused with 1.5\% low-gelling agarose (Sigma) and $1 \mathrm{mg} / 5 \mathrm{ml}$ BSA-rhodamine in Leibovitz's L-15 medium. Brains were removed, immediately chilled to $4^{\circ} \mathrm{C}$ to solidify the agarose, and coronal sections cut on a vibratome $(300 \mu \mathrm{m})$ and placed in continuously oxygenated artificial CSF medium [containing (in mM): $120 \mathrm{NaCl}, 3 \mathrm{KCl}, 2$ $\mathrm{CaCl}_{2}, 1.3 \mathrm{MgSO}_{4}, 25 \mathrm{NaHCO}_{3}, 10$ glucose, 5 BES ( $N, N$-bis $(2-$ hydroxyethyl)-2-aminoethanesulfonic acid)] maintained in a $34^{\circ} \mathrm{C}$ thermocontrolled flow $(2.5 \mathrm{ml} / \mathrm{min})$ chamber. Time-lapse images were captured every $15 \mathrm{~min}$ for a total period of $60-150 \mathrm{~min}$ on a FluoView $\mathrm{X}$ confocal microscope at a high scan speed to minimize light exposure to the cells. To measure the speed of cells, the $x$ and $y$ coordinates of the center of every moving cell were plotted in CorelDraw and the distance traveled calculated using the Pythagorean theorem. The total migratory rate was calculated by summating the distances traveled between each frame divided by the total imaging time. Data are given as mean \pm SEM.

\section{Results}

In adults, new neurons derived from the SVZ migrate from the center of the $\mathrm{OB}$ to their final destinations in outer layers in the absence of anatomically distinct glial scaffolds. To investigate vascular scaffolds present in the adult brain, we labeled migrating SVZ progenitors by injecting CTG in the SVZ of P21 mice (De Marchis et al., 2001) and, 3 d later, labeled blood vessels by perfusion with a gelatin/BSA-rhodamine mix. The gelatin rapidly solidifies within the vessels and the rhodamine allowed visualization of the lumen of all blood vessels from large to small capillaries (Fig. 1 $A-C$ ). CTG-labeled cells were frequently observed closely associated with blood vessels in the granule cell layer (GCL) (Fig. 1D) and, in may cases, the migrating cell leading process extended or twisted around small vessels (Fig. $1 E-H$ ). The olfactory bulb is highly vascular with vessels oriented in different directions, including many radially oriented as well as tangentially oriented (average vessel orientation in the bulb was $42 \pm$ $4^{\circ}$, with $0^{\circ}$ representing a tangential orientation and $90^{\circ}$ a radial orientation). CTG-labeled cells had a slight tendency to associate preferentially with radially oriented capillaries (average orientation for vessels with migrating cells was $52 \pm 3^{\circ} ; p<0.05 ; n=43$ cells), although CTG-positive cells could be found on vessels with different orientations.

To quantify the association of CTG-positive cells with vascular elements we measured the $x, y$, and $z$ positions of 148 randomly selected CTG cells and calculated the distance to the nearest blood vessel in three dimensions, yielding a distance/density
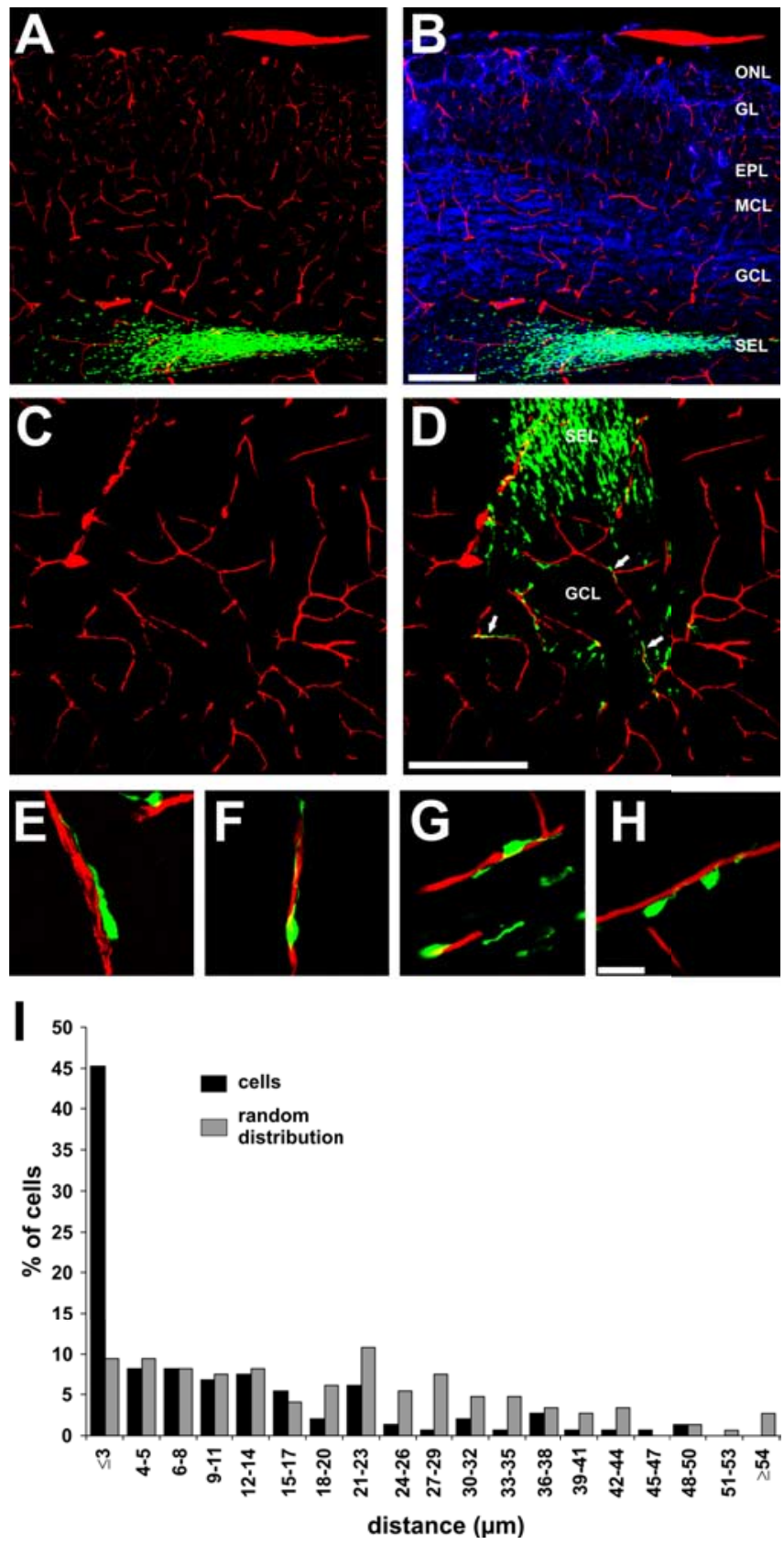

Figure 1. SVZ-derived migratory cells associated with blood vessels. $A-B$, Coronal sections of the OB showing CTG-positive cells (green) and blood vessels $(\boldsymbol{A}$; lumen tetramethylrhodamine isothiocyanate fill; red) counterstained with DAPI ( $\boldsymbol{B}$; blue). $\boldsymbol{C}-\boldsymbol{D}$, Coronal section of P24 mouse $\mathrm{OB}$ showing blood vessels $(\boldsymbol{C} ;$ red); neuronal precursors (green) exiting the SEL and entering the $\mathrm{GCL}$ associate with blood vessels ( $\boldsymbol{D}$; arrows). $\boldsymbol{E}-\boldsymbol{H}$, Higher-magnification photographs of coronal OB sections showing (Tb-labeled cells associated to blood vessels. I, Frequency distribution graph of the number of CTG-positive cells (abscissa) versus distances from the blood vessels (ordinate). The number of (TG-positive cells closer than $3 \mu \mathrm{m}$ is significantly different from a random distribution (MANOVA, ${ }^{*} p<0.001$ ). Scale bars: (in $\left.\boldsymbol{B}\right) \boldsymbol{A}, \boldsymbol{B}, 200 \mu \mathrm{m}$; (in D) C, D, $200 \mu \mathrm{m}$; (in $\boldsymbol{H}) \boldsymbol{F}-\boldsymbol{H}, 20 \mu \mathrm{m}$. MCL, Mitral cell layer; ONL, olfactory nerve layer.

histogram of cell position relative to the nearest blood vessels. The mean distance between cell and vessel was $9.5 \pm 1.0 \mu \mathrm{m}(n=$ 148 cells) (Fig. $1 J$ ). To test whether the association of the migrating cells with blood vessels is simply chance because of a high density of vessels in the bulb, the distribution of CTG cells was compared with a random distribution. This analysis showed that random points in these sections are on average $20.4 \pm 1.2 \mu \mathrm{m}$ $(n=148)$ away from a vessel, compared with the average for CTG 
cells of $9.5 \pm 1.0 \mu \mathrm{m}$. This analysis shows that cells are much closer to vessels than random chance would predict $(p<$ 0.001 ) (Fig. $1 J$ ), with $45 \%$ of CTGpositive cells distributed $\leq 3 \mu \mathrm{m}$ from the vessels (Fig. 1I). The remaining 55\% of cells are distributed at different distances from vessels and likely include differentiated cells that have already reached their final destination in the GCL, cells that have just started to migrate out of the subependymal layer and not yet encountered a vessel, and migrating cells independent of vessels.

Diffusible molecules, even oxygen, released from the blood vessels could be attractive cues for the migrating cells. If this is the case, the diffusion of these putative factors should result in a gradient of CTG cells migrating toward blood vessels. However, our results show that there is a significant difference only in the proportion of cells in contact with the vessel $(<3$ $\mu \mathrm{m})$ and no difference from a random distribution at distances $>3 \mu \mathrm{m}$ (Fig. $1 I$ ). Thus, we conclude that interaction between blood vessels and migrating cells is likely a contact-mediated process rather than attraction by secreted molecules.

Previous findings suggest there are precursors in the early SVZ and RMS that can give rise to neurons and glia (Aguirre and Gallo, 2004). To investigate whether the cells migrating along vessels were preferentially glial or neuronal, we performed immunohistochemistry with GFAP, an astrocyte marker (Fig. $2 B$ ). None of the migrating CTG-positive cells in the olfactory bulb expressed GFAP ( $n=346$ cells; $2-4 \mathrm{~d}$ after injection). To further investigate whether SVZ precursors can differentiate into astrocytes in the OB, SVZ precursors were labeled with FG and immunostained for GFAP 30 d later. Only 1\% of FG-labeled SVZderived cells in the bulb ( 1 cell of 109 examined) expressed GFAP, suggesting that only a very small population of SVZ precursors differentiate into astrocytes (data not shown).

To investigate whether CTG-labeled cells associated with the blood vessels are mature neurons, we performed immunohistochemistry for NeuN, a mature neuronal marker. None of the labeled migratory cells associated with the blood vessels labeled with NeuN ( $n=150)$ (data not shown). In our previous studies, we showed that in a transgenic line of mice expressing green fluorescent protein (GFP) under the control of the glutamic acid decarboxylase $65 \mathrm{kDa}$ (GAD65) promoter, there was extensive expression of the neuronal GAD65 gene during migration. In these mice, cells along the entire length of the RMS and in the OB express GAD65-GFP (De Marchis et al., 2004). We also observed GAD65-GFP positive migratory cells associated with blood vessels (Fig. 2A) indicating that neuronal migratory cells associate with blood vessels.

The association between SVZ-derived cells and vessels could involve neuroblast to endothelial cell, neuroblast to astrocyte, or neuroblast to extracellular matrix basement components surrounding the vessels. We rarely observed GFAP-positive processes between CTG-labeled cells and blood vessels (Fig. 2B); however, GFAP immunohistochemistry does not show very fine astrocytic processes even with confocal microscopy. To improve our spatial resolution in the perivascular compartment, we performed immunoelectron microscopy analysis of the perivascular space containing migratory cells ( $n=5$ different cells). To confirm the identity of migratory cells, they were labeled with $\mathrm{CTb}$, reacted with $\mathrm{DAB}$, and the same cell identified under both light microscopy (Fig. 2C) and electron microscopy $(D, E)$. All blood vessels in the olfactory bulb exhibited a thin astrocyte process sheet surrounding the vessel; in places, these processes were as thin as $20-50 \mathrm{~nm}$ (Fig. $2 \mathrm{E}$ ). The $\mathrm{CTb} / \mathrm{DAB}$ reaction product in migratory cells was typically seen concentrated in punctuate lysosome vesicles. None of the SVZ-derived cells directly contacted endothelial cells or endothelial cell basement membranes. Instead, these cells were typically superficial to the astrocyte processes and separated by a thin region of ECM (Fig. 2E). This suggests that cells migrating along blood vessels are primarily interacting with the ECM surrounding astrocyte end feet and, in some regions, by direct astrocyte contact.

Together, these data indicate that a large number of SVZderived cells associate with vessels in the GCL trough an ECMastrocyte end foot interaction. The close association of CTGlabeled cells with blood vessels suggests migration along the vessels; however, a similar statistical association might be obtained if there was merely heightened survival of cells close to the vessel, similar to hypothesizes in the RMS and hippocampus (Palmer et al., 2000). To determine whether the association of cells with blood vessels was truly related to migration, we performed double-label time-lapse confocal microscopy on coronal slices of the $\mathrm{OB}$ and followed the movement of the cells along the vessels. Because gelatin melts at $34^{\circ} \mathrm{C}$, the labeling of blood vessels for the time lapse was performed using low-gelling agarose. The 

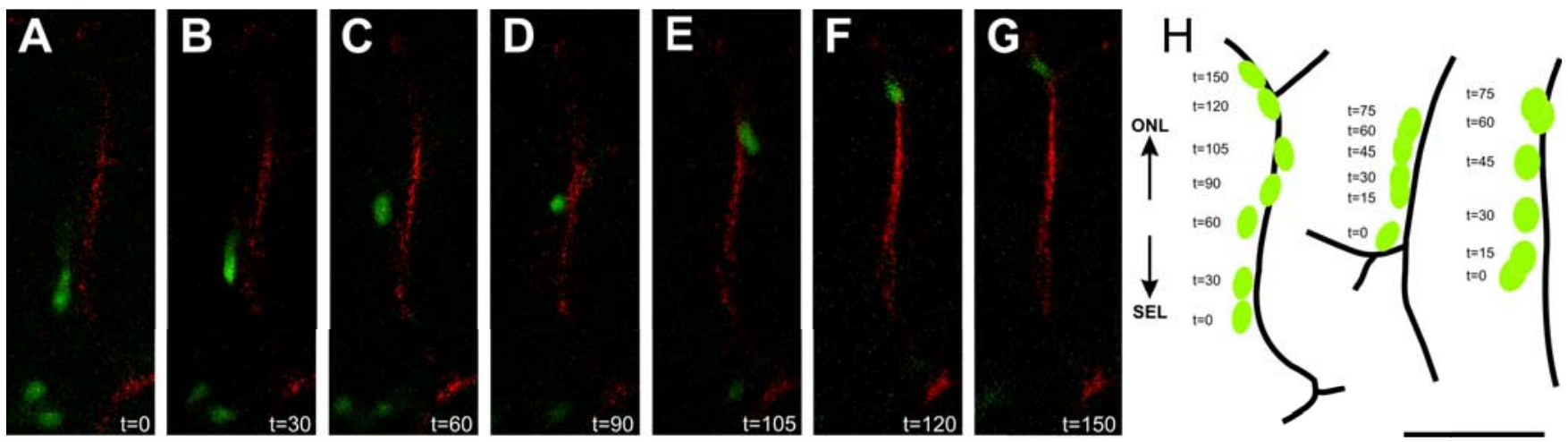

Figure 3. Double-label time-lapse confocal microscopy showing neuronal precursors migrating along the blood vessels. A-G, Photographs of a (TG-labeled neuroblast (green) and blood vessel (red) at selected time points from $t=0 \mathrm{~min}$ to $t=150 \mathrm{~min}$. $\boldsymbol{H}$, Schematic drawing of three different cells moving along vessels as a function of time. Scale bar: (in $\boldsymbol{H}) \boldsymbol{A}-\boldsymbol{H}, 50 \mu \mathrm{m}$. $0 \mathrm{NL}, 0$ lfactory nerve layer.

movement of five different cells in relation to blood vessel position was followed with images taken every 15 min for a total imaging time between 60 and $150 \mathrm{~min}$ (Fig. 3A-H). CTG-labeled migratory cells have unipolar morphology with a leading process in the direction of migration typical of migratory neurons and observed in our previous studies (De Marchis et al., 2001; Bovetti et al., 2007). Cells migrate along the vessels in a saltatory modality, alternating between higher and lower migration speed (average maximum speed $66 \pm 17 \mu \mathrm{m} / \mathrm{h}$; average minimum speed $6 \pm$ $3.5 \mu \mathrm{m} / \mathrm{h} ; n=5)$. The mean migration rate of CTG-labeled cells along a blood vessel was $33.4 \pm 5.2 \mu \mathrm{m} / \mathrm{h}(n=5)$. The migration of the CTG-positive cells was unidirectional, with all of the cells observed moving radially along vessels toward the external part of the bulb.

Our data show that cells traversing the deep GCL use blood vessels as a migratory scaffold. Do the migrating cells continue to use blood vessels all the way out to the glomerular layer (GL)? To address this question, we examined $7 \mathrm{~d}$ postinjection animals in which migratory cells are crossing the external plexiform layer (EPL). CTG-positive cells in the EPL were counted and their association to vessels evaluated as above. Only 3 of 54 cells ( $n=2$ mice) in the EPL were in direct contact with blood vessels. These data suggest that although "vasophilic migration" is an initial mechanism used by cells to detach from the subependymal layer (SEL) and move radially through the GCL, it is not required to cross the EPL.

These data demonstrate a new modality of neuroblast migration after blood vessels in the young adult olfactory bulb.

\section{Discussion}

OB neuronal progenitors are born in the SVZ and migrate to the olfactory bulb along a pathway referred to as the RMS. After reaching the bulb, these cells migrate radially to various layers of the OB, where they differentiate in different classes of interneurons. The mechanisms regulating this radial migration into the OB are poorly understood. During development, immature neurons use the processes of radial glia cells as a guide to migrate radially and reach their final destination in many brain structures. Although radial glia are present in developing olfactory bulb and are hypothesized to play a role in the formation of the bulb (Bailey et al., 1999; Puche and Shipley, 2001), they disappear in early postnatal and are absent in adult (Bailey et al., 1999; Puche and Shipley, 2001).

We described previously the behavior of radially migrating cells in the adult $\mathrm{OB}$, which exhibit a long leading process and a shorter trailing process typical of migrating cells. These cells move radially in a saltatory manner with bursts of high speed followed by periods of slower speed (Bovetti et al., 2007). In this study, we show a modality of radial migration in the adult bulb in which neuroblasts use the blood vessels as a migratory scaffold. Neuronal progenitors in the GCL of the OB are closely associated with blood vessels, have leading/trailing processes, and extend the leading process along or, in some cases, around a vessel. Time-lapse confocal microscopy demonstrates that neuroblasts migrate along the vessels in a saltatory manner, altering periods of slow and high speeds. The migratory behavior (average speed, saltatory frequency, maximum, and minimum speeds) of cells associated with vessels was identical to the means of our previous report (Bovetti et al., 2007)

Most adult SVZ-derived cells (>95\%) differentiate into GABA-containing granule cells, whereas the rest differentiate into periglomerular cells in the glomerular layer (Luskin, 1998). Our data show that $45 \%$ of CTG-positive cells associate to vessels in the GCL. Because this frequency of cell-blood vessel distances were calculated choosing random cells, this likely includes differentiated cells that have already reached their final destination in the GCL, as well as cells that just began radial migration. Therefore, we may underestimate the actual number of undifferentiated migrating cells that associate with a vessel at some point during their migration. Alternatively, vessels may be a "path of least resistance" to cell movement, resulting in more cells migrating along a vessel when it is present with some cells migrating in the absence of the vessel scaffold. It is clear that by the time migratory cells enter the EPL they are no longer specifically associated with blood vessels. Possibly the radially oriented apical dendrites of mitral cells provides a structural scaffold for crossing the EPL.

Transplanted cells in the adult brain often migrate long distances from the injection location and, based on our results, may use blood vessels to traverse to other locations in the brain. Indeed, previous studies characterizing the dynamics of glioma cell migration in brain showed that transplanted rat glioma cells infiltrated the neonatal rat brain by migrating along the abluminal surface of blood vessels (Farin et al., 2006). Farin et al. (2006) show that transplanted glioma cells are localized in either multicellular clusters or individual cells distributed along host blood vessels and, through time lapse microscopy, they confirmed that the cells were migrating along vessels. Similar to glial and neuronal progenitors, these glioma cells display a unipolar morphology 
with the major process closely aligned with blood vessels, they migrate in a salutatory manner and frequently turn or reverse direction, suggesting that they are responding to local guidance cues (Farin et al., 2006). Here, we extend these anatomical observations with evidence that normal neuroblasts migrating de novo through the adult brain use blood vessels as a scaffold. Radially migrating cells in the adult $\mathrm{OB}$ rarely migrate in the wrong direction $(<1 \%)$ (Bovetti et al., 2007) and we did not observe any cell migrating on vessels moving in the opposite direction. The mechanisms governing direction choice in the bulb are unknown; however, several secreted molecules such as netrin-1, prokineticin2, and glial cell-derived neurotrophic factor have been suggested to have a chemoattractive role for $\mathrm{OB}$ precursors and could provide center-to-periphery cues (Murase and Horwitz, 2002; Ng et al., 2005; Paratcha et al., 2006).

What might attract migrating cells to blood vessels? In our time-lapse experiments, blood-borne diffusible attractants would have been washed from the vessel suggests a contactmediated rather than diffusible cue. The electron microscopy analysis confirmed this hypothesis, showing that neuroblasts associate with extracellular matrix components surrounding astrocytic end feet. This is different from previous glioma studies showing direct association of cells with the abluminal surface of the vessels (Farin et al., 2006). It is possible that the glioma has glial cues similar to perivascular astrocytes, which permit close association to the endothelial cells. The extracellular matrix plays an important role in cell migration, providing permissive or inhibitory substrates to migration (Ono et al., 1994), and inhibition of matrix metalloproteinases, the enzymes responsible of extracellular matrix modification, inhibits both tangential and radial migration in the RMS and in the $\mathrm{OB}$ of postnatal and adult mice (Bovetti et al., 2007). Moreover, previous studies reported that reelin and tenascin-R, two extracellular matrix molecules, are involved in cell detachment and radial migration into the $\mathrm{OB}$ (Hack et al., 2002; Saghatelyan et al., 2004). Tenascin or other ECM elements might be produced by perivascular astrocytes and govern neuroblast migration out of the RMS along blood vessels. Although additional studies are needed to understand the mechanisms governing neuroblast-blood vessel interactions and migration and the molecules involved in these processes, here we provide the first evidence that neuronal precursors follow the blood vessels to reach their final destination, interaction with perivascular astrocytes end feet, revealing a novel role of brain vasculature and opening a new and unexplored field in adult neuronal migration.

\section{References}

Adams JC (1981) Heavy metal intensification of DAB-based HRP reaction product. J Histochem Cytochem 29:775.

Aguirre A, Gallo V (2004) Postnatal neurogenesis and gliogenesis in the olfactory bulb from NG2-expressing progenitors of the subventricular zone. J Neurosci 24:10530-10541.

Bailey MS, Puche AC, Shipley MT (1999) Development of the olfactory bulb: evidence for glia-neuron interactions in glomerular formation. J Comp Neurol 415:423-448.

Bovetti S, Bovolin P, Perroteau I, Puche AC (2007) SVZ-derived neuroblast migration to the olfactory bulb is modulated by matrix remodeling. Eur J Neurosci 25:2021-2033.

De Marchis S, Fasolo A, Shipley M, Puche A (2001) Unique neuronal tracers show migration and differentiation of SVZ progenitors in organotypic slices. J Neurobiol 49:326-338.

De Marchis S, Temoney S, Erdelyi F, Bovetti S, Bovolin P, Szabo G, Puche AC (2004) GABAergic phenotypic differentiation of a subpopulation of subventricular derived migrating progenitors. Eur J Neurosci 20:1307-1317.

Farin A, Suzuki SO, Weiker M, Goldman JE, Bruce JN, Canoll P (2006) Transplanted glioma cells migrate and proliferate on host brain vasculature: a dynamic analysis. Glia 53:799-808.

Gasser UE, Hatten ME (1990a) Neuron-glia interactions of rat hippocampal cells in vitro: glial-guided neuronal migration and neuronal regulation of glial differentiation. J Neurosci 10:1276-1285.

Gasser UE, Hatten ME (1990b) Central nervous system neurons migrate on astroglial fibers from heterotypic brain regions in vitro. Proc Natl Acad Sci USA 87:4543-4547.

Hack I, Bancila M, Loulier K, Carroll P, Cremer H (2002) Reelin is a detachment signal in tangential chain-migration during postnatal neurogenesis. Nat Neurosci 5:939-945.

Lois C, Garcia-Verdugo JM, Alvarez-Buylla A (1996) Chain migration of neuronal precursors. Science 271:978-981.

Luskin MB (1998) Neuroblasts of the postnatal mammalian forebrain: their phenotype and fate. J Neurobiol 36:221-233.

Murase S, Horwitz AF (2002) Deleted in colorectal carcinoma and differentially expressed integrins mediate the directional migration of neural precursors in the rostral migratory stream. J Neurosci 22:3568-3579.

Ng KL, Li JD, Cheng MY, Leslie FM, Lee AG, Zhou QY (2005) Dependence of olfactory bulb neurogenesis on prokineticin 2 signaling. Science 308:1923-1927.

Ono K, Tomasiewicz H, Magnuson T, Rutishauser U (1994) N-CAM mutation inhibits tangential neuronal migration and is phenocopied by enzymatic removal of polysialic acid. Neuron 13:595-609.

Palmer TD, Willhoite AR, Gage FH (2000) Vascular niche for adult hippocampal neurogenesis. J Comp Neurol 425:479-494.

Paratcha G, Ibanez CF, Ledda F (2006) GDNF is a chemoattractant factor for neuronal precursor cells in the rostral migratory stream. Mol Cell Neurosci 31:505-514.

Peretto P, Giachino C, Aimar P, Fasolo A, Bonfanti L (2005) Chain formation and glial tube assembly in the shift from neonatal to adult subventricular zone of the rodent forebrain. J Comp Neurol 487:407-427.

Puche AC, Shipley MT (2001) Radial glia development in the mouse olfactory bulb. J Comp Neurol 434:1-12.

Saghatelyan A, de CA, Schachner M, Lledo PM (2004) Tenascin-R mediates activity-dependent recruitment of neuroblasts in the adult mouse forebrain. Nat Neurosci 7:347-356.

Toida K, Kosaka K, Heizmann CW, Kosaka T (1998) Chemically defined neuron groups and their subpopulations in the glomerular layer of the rat main olfactory bulb: III. Structural features of calbindin D28Kimmunoreactive neurons. J Comp Neurol 392:179-198.

Wichterle H, Garcia-Verdugo JM, Alvarez-Buylla A (1997) Direct evidence for homotypic, glia-independent neuronal migration. Neuron 18:779-791.

Yang P, Baker KA, Hagg T (2005) A disintegrin and metalloprotease 21 (ADAM21) is associated with neurogenesis and axonal growth in developing and adult rodent CNS. J Comp Neurol 490:163-179. 